\title{
AN ESR AND X-RAY DIFFRACTION STUDY OF A FIRST-ORDER PHASE TRANSITION IN CdPS 3
}

\section{E. Lifshitz and A.H. Francis}

Department of Chemistry, The University of Michigan, Ann Arbor, Michigan 48109

and

Roy Clarke

Department of Physics, The University of Michigan, Ann Arbor, Michigan 48109

(Received 8 oct. 1982 by G. Burns)

The ESR spectra of $\mathrm{Mn}^{+2}$ doped single crystals of $\mathrm{CdPS}_{3}$ indicate a

first-order phase transition (monoclinic to orthorhombic) at ca. $260 \mathrm{~K}$.

This is confirmed directly by an X-ray diffraction examination of the

doped and undoped crystals at various temperatures.

\section{Introduction}

The transition metal chalcogenophosphates form a series of isomorphous compounds with the general chemical formula $\mathrm{MPX}_{3}$ where $M$ is a row-four transition metal and $X$ is either $S$ or Se. 1 A full crystal structure has been reported only for the room-temperature iron compound of sulfur. 2,3 However, the room-temperature 1 attice parameters and crystal space group have been determined for all compounds in the series.1 At ambient temperature, the transition metal sulfides are reported to be either monoclinic or trigonal and the selenides, trigonal.

These materials all crystallize in a layered structure with $\mathrm{planes}$ of hexa-coordinated metal atoms sandwiched between planes of hexagonally packed chalcogen atoms. The phosphorus atom-pairs occupy sites in the metal atom plane. The metal atom coordination geometry is trigonally-distorted octahedral in both the monoclinic and the trigonal forms. The macroscopic structure is built from repeated layers of the $X-M-X$ sandwich structure held together by weak van der Waals forces between the adjacent chalcogenide planes.

The layered structure of the $\mathrm{MPX}_{3}$ compounds is similar to that of the more extensively studied transition metal dichalcogenides, $M X_{2} \cdot 4$ The $\mathrm{MPX}_{3}$ structure may be viewed as the $M X_{2}$ structure with $1 / 3$ of the metal replaced by a $\mathrm{P}_{2}$ atom-pair. ${ }^{, 5}$ The effect of intercalation on the charge transport properties of $\mathrm{MX}_{2}, 8$

intercalation compounds has been extensively investigated. Relatively little research in this area has been reported for the intercalation compounds of $\mathrm{MPX}_{3}$ materials, to which end the present study is a prelude. Extensive investigations of structural phase transitions in the quasi two-dimensional transition metal dichalcogenides have been reported, while there have been no reports to date of similar transitions in the iso-structural chal cogenophosphates.

It is the purpose of the present communication to report on the observation and characterization of a first-order phase transition in the compound $\mathrm{CdPS}_{3}$. The phase transition was observed in single crystals using $x$-ray diffraction techniques and ESR spectroscopy of $\mathrm{Mn}^{+2}$ substitutional impurity centers.

Experimental

$\mathrm{CdPS}_{3}$ was synthesized from a powdered mixture containing stoichiometric amounts of $\mathrm{CdS}$, phosphorus (red) and sulfur. Samples for use in the ESR studies were doped with 1 at. $\% \mathrm{Mn}$.

Large single crystals $\left(5 \times 5 \times 0.1 \mathrm{~mm}^{3}\right)$ were grown from the vapor phase 3 in evacuated vycor tubes. The tubes were placed in a two-zone vertical tube furnace. The material was sublimed from the hotter to the cooler zone. A temperature gradient of $943 \mathrm{~K}+873 \mathrm{~K}$ produced excellent crystal growth in approximately one week.

Elemental analysis of $\mathrm{CdPS}_{3}$ crystals prepared by this method was in agreement with the empirical formula (weight $\% c d=45.89$, $\% p=12.24$, $\% S=41.83$ ). For ESR investigation, crystal's were oriented conoscopically by identifying the optic axes in the ab crystal plane. The b-axis could be identified from the crystal morphology. Single crystals were mounted on a rotating sample stage attached to the microwave cavity of a Brücker model ER200E X-band ESR spectrometer and spectra were recorded as a function of the angle of rotation about the crystallographic $a, b$ and $c^{\star}(a \times b)$ axes of the room-temperature monoclinic structure. The temperature of the crystal could be varied using a Varian Model 4257 variable temperature accessory modified to fit the microwave cavity.

For X-ray diffraction studies, small single crystals were mounted on a Huber model 100 Weissenberg camera fitted with a liquid nitrogen cooling attachment (stability $\pm 0.2 \mathrm{~K}$ ). The crystal was oscillated around various crysta1lographic axes in the ab plane in order to pick up reflections sensitive to the observed structural distortion, in this case the monoclinic angle, $B$. A small number of such reflections were isolated by appropriate choice of oscillation range and zero-layer Weissenberg screens. In order to record changes occurring at 
the phase transition, the X-ray film was translated stepwise (every $\sim 10$ minutes) as the temperature was varied in $3 \mathrm{~K}$ steps. A $1 \mathrm{~kW}$ sealed-tube source of $\mathrm{Cu} K$ radiation was used throughout. In this way, a rather striking record of variations in lattice parameters across the transition is obtained. Further details of the method appear in Ref. 9.

\section{Results}

The angular dependence of the ESR spectrum of $\mathrm{Mn}^{+2}$ centers in CdPS 3 was recorded over the temperature range $140 \mathrm{~K}$ to $500 \mathrm{~K}$. Above $300 \mathrm{~K}$, the spectrum was characteristic of a single manganese site (spectrum A, Figure 1); however, as the crystal was cooled below about $220 \mathrm{~K}$, all the lines in the spectrum diminished in intensity and a second set of lines (spectrum B, Figure 1) appeared which gradually became dominant at the lowest temperatures attained. The fractional intensity of the high temperature spectrum is plotted as a function of temperature in Figure 1 where the small arrows indicate the direction of temperature change during measurement. The observed intensity variations are reproducible and exhibit substantial hysteresis.

The marked behavior shown in Figure 1 is also seen, in Figure 2, to be accompanied by strong discontinuities in at least one of the lattice parameters over approximately the same temperature range, confirming that a first-order structural transition is responsible for the observed changes in the ESR spectrum. On cooling from the room-temperature monoclinic phase (see Figure 2(b)) a new set of $X$-ray reflections appear at $T^{+}=248 \mathrm{~K}$. These reflections are indexed on the basis of an orthorhombic cell with the same contents as the original monoclinic cel1. Ful1 Weissenberg data of the $a^{\star} c^{\star}$ plane, taken at $T=215 \mathrm{~K}$, confirms that indeed $B$ changes discontinuously from $107^{\circ}$, its value at room temperature, to precisely $90^{\circ}$ at the phase transition. In contrast, only small changes in the other lattice parameters determining interplanar spacings are observed at the phase transition (see Table 1). On heating (Figure $2(a)$ ), the transition is seen to be reversible, occurring at $\mathrm{T}^{\uparrow}=264 \mathrm{~K}$.

\section{Discussion}

The data presented in Figures 1 and 2 suggest the existence of a first-order phase transition centered at about $260 \mathrm{~K}$. Between approximate1y 220 and $290 \mathrm{~K}$ both phases co-exist as is clear from the multiple site nature of the ESR spectrum, The angular dependence of the ESR spectrum of $\mathrm{Mn}^{+2}$ centers in CdPS 3 obtained both at ambient temperatures and below, could be accurately reproduced by theoretical calculation with the following spin-Hamiltonian, which is suitable for orthorhombic or lower symmetry 10

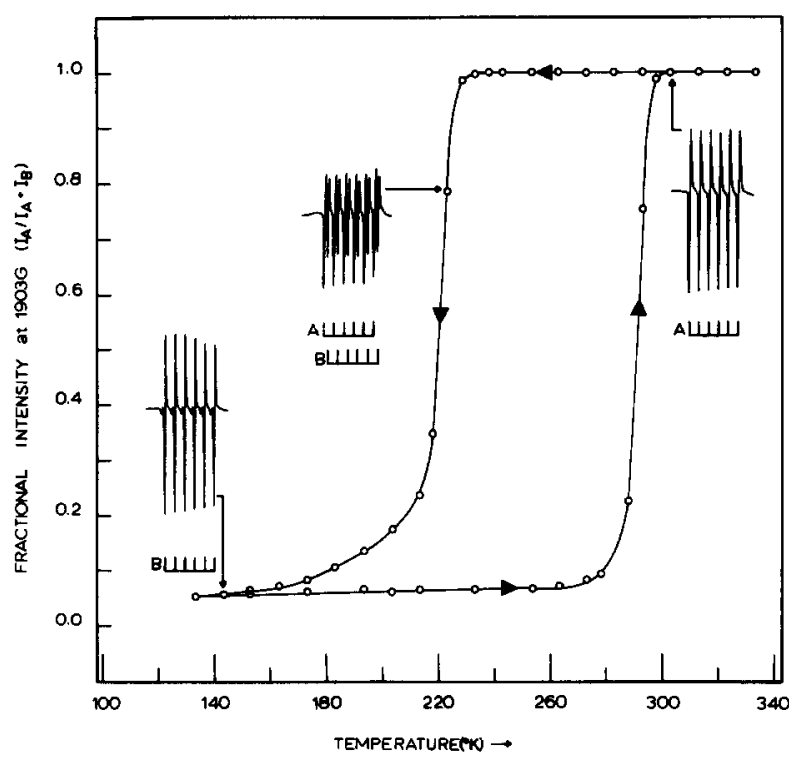

Figure 1: Fractional Intensity $\left(\frac{I_{A}}{I_{A}+I_{B}}\right)$ of the ESR spectrum of $\mathrm{Mn}(\mathrm{II})$-doped $\mathrm{CdPS}_{3}$. Insets show $A$ and $B$ phase spectra and arrows indicate direction of cooling and heating.

parts of the fine structure (zero field splitting), the fourth term represents the cubic part of the crystal field and the last term represents the nuclear Zeeman (hyperfine) interaction $S=I=5 / 2$ for $M(I I)\left(d^{5}\right)$. It was assumed that the electronic Zeeman interaction, the zero-field interaction and the hyperfine interaction all have the same set of principal axes $(x, y$ and $z)$. The $y$-axis of the spin-Hamiltonian was chosen parallel to the two-fold crystallographic axis (b-axis) of the room temperature monoclinic lattice. The $x$ and $z$ principal axes then lie in the crystallographic ac plane perpendicular to $\vec{b}$.

The angular variation of the ESR spectra was analyzed by numerical diagonalization of the matrix of the spin-Hamiltonian, Equation 1. The parameters of the spin-Hamiltonian for both the room-temperature and low-temperature phases of $\mathrm{CdPS}_{3}$ are given in Table 1. These were obtained by using an iterative procedure to fit the theoretical spectrum to the experimental data.

The fact that the crystal field parameters ( $D, E$ and $a)$ all have non-zero values, and that $g$ shows a small anisotropy for the room temperature phase indicates that $\mathrm{Mn}^{+2}$, which substitutionally replaces $\mathrm{Cd}^{+2}$, has nearly axial symmetry with a slight rhombic distortion.11,12 This result is consistent with the proposed monoclinic room-temperature structure of $\mathrm{CdPS}_{3}, 1,3$ The zero

$$
\begin{aligned}
H_{S} & =B_{0} \cdot H \cdot g \cdot S+D\left[S_{z}^{2}-1 / 3 S(S+1)\right]+E\left(S_{x}^{2}-S_{y}^{2}\right)+1 / 6 a\left[S_{x}^{4}+S_{y}^{4}+S_{z}^{4}\right. \\
& \left.-1 / 5 S(S+1)\left(3 S^{2}+3 S-1\right)\right]+A_{z z} S_{z} I_{z}+A_{x x} S_{x} I_{x}+A_{y y} S_{y} I_{y} \cdot
\end{aligned}
$$

where the first term represents the electronic Zeeman interaction, the second and third terms represent, respectively, the axial and rhombic value obtained for the rhombic distortion parameter in the low-temperature phase suggests a rigorously orthorhombic lattice. This is 
Table 1

Symmetry Monoclinic $(300 \mathrm{~K})$ Orthorhombic (143 K)

$\begin{array}{lcc}D & -365 \pm 2 & -353 \pm 2 \\ E & -6 \pm 1 & 0 \\ A_{X X} & -69.2 \pm 1 & -69 \pm 2 \\ A_{y y} & -69.4 \pm 1 & -69 \pm 2 \\ A_{Z Z} & -70.5 \pm 1 & -70 \pm 2 \\ g_{X X} & 2.006 \pm 0.002 & 2.005 \pm 0.001 \\ g_{y y} & 2.007 \pm 0.002 & 2.005 \pm 0.001 \\ g_{Z Z} & 2.001 \pm 0.002 & 2.002 \pm 0.001 \\ a^{\prime} & 0.7 \pm 0.2 & 0.3 \pm 0.2 \\ \vec{a} & 6.17 \AA \star & 6.18 \pm 0.03 \AA \star \star \\ \vec{b} & 10.67 \AA & 10.67 \pm 0.05 \AA \\ \vec{C} & 6.82 \AA & 6.40 \pm 0.02 \AA \\ B & 107.1 & \end{array}$

Note: The units of $D, E, A_{i j}$ and a are $10^{-4} \mathrm{~cm}^{-1}$.

*Ref. 1 .

**This study.
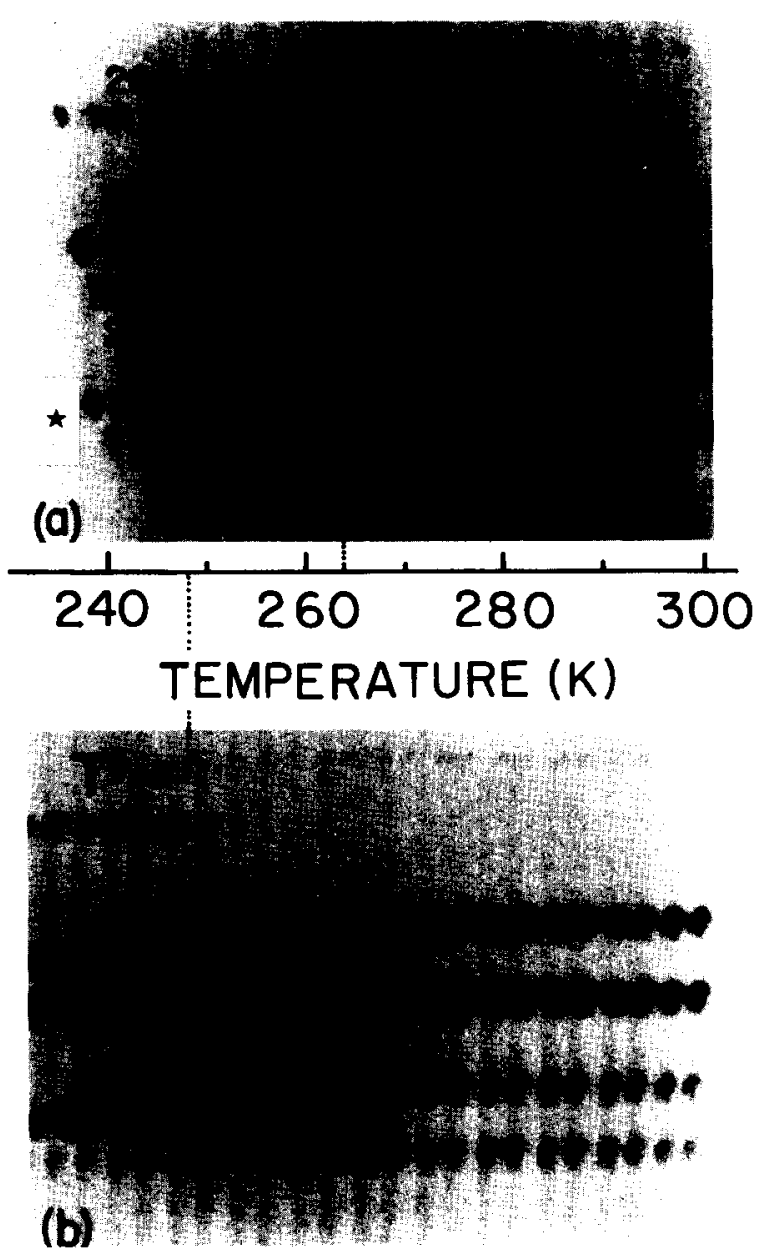

Figure 2: Sequential $X$-ray oscillation exposures for a single crystal of undoped $\mathrm{CdPS}_{3}$, a) on heating, b) on c00ling. * denotes reflections formed by the $\beta$-component of the radiation. The indexed spots are from $\mathrm{Cu} K \bar{\alpha}$. confirmed directly by $X$-ray diffraction in the low-temperature phase. The establishment of a mirror plane parallel to $a^{*}$ at the transition, together with systematic absences of the type $h+k \neq 2 n$, identifies Cmm2 and Cmm as possible low-temperature space groups, the former being distinguished by non-centrosymmetry. At this point, a full structural determination has not been carried out.

The thermal hysteresis of $T^{+}-T^{+}=16 \mathrm{~K}$, as shown in Figure 2, is considerably smaller than the value of $40 \mathrm{~K}$ given by the ESR results in Figure 1. This difference is due to the sensitivity of the ESR technique to small amounts of the minor coexisting phase which occurs, respectively, at the upper and lower edges of the hysteresis loop in Figure 1 . This coexistence is al so evident, but less so, in Figures $2(a)$ and 2(b).

Lattice parameter measurements, listed in Table 1, indicate that the pseudo-hexagonal arrangement of the ab layer is well preserved through the transition (i.e., $b \approx a \sqrt{3}$ ) and that the structural transition involves principally a sliding motion of these layers, aided by the weak van der Waals bonding. This mechanism is al so consistent with the small (2\%) decrease in the interlayer spacing at the transition.

There are two unusual features of the phase transition in $\mathrm{CdPS}_{3}$ which should be mentioned. Firstly, the change in monoclinic angle $\left(\Delta \beta=17.1^{\circ}\right)$ at the transition is surprisingly large; especially since this implies relative displacement of adjacent layers by as much as $2 \AA$. Secondly, the structural symmetry appears to increase on cooling, with the loss of the monoclinic-phase unique axis and the establishment of at least one extra mirror plane in the orthorhombic phase. This is the reverse of the familiar cases in which the symmetry is broken by the appearance of an order parameter in the low-temperature phase. A similar transition has been reported 13 in the monoclinic layer compound $\beta-\mathrm{MoTe}_{2}$, the low-temperature phase again being orthorhombic. The reason for the instability of these monociinic compounds is not understood at 
this time. There is no evidence for charge density waves or incommensurate behavior in either of these monoclinic structures in the form of electron diffraction satellites.

\section{Conclusion}

The ESR spectra of $\mathrm{Mn}^{+2}$ doped $\mathrm{CAPS}_{3}$ single crystals and the $x$-ray diffraction of doped and undoped crystals are consistent with a first-order structural phase transition from the room-temperature monoclinic lattice to a low-temperature orthorhombic lattice occurring at about $250 \mathrm{~K}$. The transition is reversible and hysteretic and involves considerable sliding of adjacent layers across the van der Waals gap.

We have also examined the ESR spectra of the $\mathrm{Mg}$ and $\mathrm{Zn}$ compounds and have found no evidence of a phase transition in the temperature range $140-500 \mathrm{~K}$. A preliminary analysis of the angular dependence of the ESR spectra of these compounds indicates that the lattices are orthorhombic at room temperature. Previous $x$-ray diffraction studies of these materials at room temperature have indicated a monoclinic lattice. Thus, it is likely that the $\mathrm{Mg}$ and $\mathrm{Zn}$ chalcogenophosphates exhibit both monoclinic and orthorhombic modifications.

Acknowledgement--This work was supported by National Science Foundation Low Temperature Physics Grant, DMR 8207449 (R.C.), and by the donors of the Petroleum Research Fund administered by the American Chemical Society.

\section{References}

1. W. Klingen, R. Ott, H. Hahn, Z. anorg. allg. Chemie, 396, 271 (1973).

2. W. Klingen, G. Eulenberger, H. Hahn, Z. anorg. allg. Chemie 401, 97 (1973).

3. B. Taylor, J. Steger, A. Wold, J. Solid State Chem. 7, 461 (1973).

4. J.A. Wilson, F.J. DiSalvo, S. Mahajan, Adv. Phys. 24, 117 (1975).

5. M. Lazzari, L.P. Biellie, B. Rivolta, An. di Chim. 71, 481 (1981).

6. R. Clement, J.C.S. Chem. Comm. 13, 647 (1980).

7. F.R. Gamble, Science (NY) 174, 493 (1971).
8. F.J. DiSalvo, J.A. Wilson, B.G. Bagley, J.V. Waszczak, Phys. Rev. B 12, 2220 (1975).

9. R. Clarke, R.E. Morley, J. Appl. Crystal1. 9, 481 (1976).

10. V.K. Jain, P. Venkateswarlu, J. Phys. C, Solid State Phys. 12, 865 (1979).

11. J. Boerio-Goates, E. Lifshitz A.H. Francis, Inorg. Chem. 203019 (1981).

12. E. Lifshitz, A.H. Francis, J. Phys. Chem., to be published.

13. R. Clarke, E. Marseglia, H.P. Hughes, Phil. Mag. B 38, 121 (1978). 